J. Perinat. Med. 29 (2001) 55-59

\section{Chlamydia trachomatis infection and the risk of perinatal mortality in Hungary}

\author{
Tibor Nyári ${ }^{1}$, Mark Woodward ${ }^{3}$, Gyula Mészáros², János Karsai ${ }^{1}$, and \\ László Kovács ${ }^{2}$ \\ ${ }^{1}$ Department of Medical Informatics, ${ }^{2}$ Department of Obstetrics and Gynaecology, \\ University, Szeged, Hungary, ${ }^{3}$ Department of Applied Statistics of University of \\ Reading, U.K.
}

\section{Introduction}

Epidemiological evidence indicates that chlamydial infections of the genital tract are a global problem. In pregnancy, Chlamydia trachomatis may cause a wide range of serious complications, including premature delivery, ophtalmia neonatorum and neonatal pneumonia. Thus, $C$. trachomatis is a major cause of neonatal morbidity and mortality.

Premature delivery is the most important perinatal problem in Hungary [3] as Czeizel and [10] Orvos have reported. In most cases the underlying cause is not known. A breakthrough in the prevention of this problem requires more data about the causes leading to premature uterine contractions. A large number of anecdotal observations suggest that $C$. trachomatis infections may have a causative role in the origin of premature delivery $[5,7,14]$. Consequently, a multicenter survey was carried out in order to determine the prevalence and risk factors for $C$. trachomatis infection in the pregnant population in Hungary [8] as Nyári has reported. The data set of the Hungarian survey was reanalyzed in order to provide further information on the relationship between neonatal mortality and genital chlamydial infections and to estimate the frequency of these infections.

\section{Materials and methods}

\subsection{The protocol}

The study started in January 1994 and terminated in June 1995, in seven different hospitals of two regions (east and west) in Hungary. The nucleic acid hybridization method (PACE2 Gen-Probe, Medical System ${ }^{\circledR}$ ) was applied for the examination of $C$. trachomatis. The sensitivity and specificity of this method are known to be $70 \%$ and $99 \%$ respectively [6] as Newhall has reported; Bayes' theorem was applied to estimate the prevalence of the infection [11]. The envisaged sample size was calculated using Hsieh's formula for logistic regression [4]. Assuming a 5\% significance test, a power of $90 \%$, and a $5 \%$ estimated probability of the infection, this resulted in a sample size of 1550 women per region. The $C$. trachomatis-positive pregnant patients received roxithromycin treatment $(150 \mathrm{mg} \times 2$ daily 10 days). Premature uterine activity was defined as any uterine contractions before the $38^{\text {th }}$ gestational week. Low birth weight was defined as birth weight less than $2500 \mathrm{~g}$. The average unemployment rate in Hungary was $6-7 \%$. If the unemployment rate was less than $4 \%$ or greater than $8 \%$, then they were regarded as low or high, respectively.

\subsection{Data collection}

The screening was performed before delivery. The target population was all women registered as singleton pregnant with their hospital, and all of them were asymptomatic as concerns of genital infection. The C. trachomatis screening was performed at the time of admission to the hospital in labor. The sample was selected using simple 
random sampling. An interviewer-administered standardized questionnaire was completed, in which age, social and marital status, obstetrical and neonatal history data were recorded. To preserve confidentially, unique codes were used. Data management was carried out with self-developed software. All personal data were protected from illegal use.

\subsection{Data analysis}

Statistical analyses were performed with the SPSS for Windows (8.0 version) software package. To compare $C$. trahomatis infected and noninfected groups chi-square tests were employed. Odds ratios (OR), as estimators of relative risks, together with their corresponding $95 \%$ confidence intervals (CI), were computed, using unconditional multiple logistic regression, fitted by the method of maximum likelihood allowing for several possible confounding factors. A $p$ value of less than 0.05 was used to indicate a significant effect.

\section{Results}

\subsection{C. trachomatis infection}

A total of 6156 pregnant women were examined for the occurrence of $C$. trachomatis. The overall average $C$. trachomatis infection rate was $5.9 \%$, the data varying in the range $1.3-9.8 \%$ (table I.).
In case of the sensitivity of the PACE2 GenProbe test for detection of $C$. trachomatis was only $70 \%$, the estimated prevalence of C. trachomatis infection in this population could be as high as was $7.1 \%$, because the additional $30 \%$ of patients with suspected infection could have been detected. These centers were categorized into two regions based on the rate of unemployment. An average rate of unemployment occurred in the western region $(3.4 \%)$ and a significant high one $(7.2 \%)$ in the eastern region.

Certain potential risk factors for $C$. trachomatis infection were examined: age, unemployment rate and family status. Young age (less than 24 years old), unmarried status and the high unemployment rate were statistically significant predictors of the infection (table II).

There was a significant difference $(p=0.001)$ between the rate of premature uterine activity that occurred in the infected group $(8.1 \%)$, and the non-infected group (5.3\%). Premature rupture of the membranes (PROM) occurred in $21.0 \%$ of the C. trachomatis-positive group and $19.9 \%$ of those without infection.

\subsection{Perinatal mortality}

Perinatal mortality occurred in $148(2.4 \%)$ of 6156 pregnancies. There was a significantly higher $(\mathrm{p}=0.042)$ mortality rate $(4.1 \%)$ in the

Table I. Observed C. trachomatis infection rates for the centers

\begin{tabular}{lrrr}
\hline Center & $\mathrm{N}$ & $\begin{array}{l}\text { C. trachomatis } \\
\text { positive }\end{array}$ & $\begin{array}{l}\text { Observed rate } \\
\text { of infection } \\
(\%)\end{array}$ \\
\hline Western region (average rate of unemployment) & 2146 & 73 & 3.4 \\
$\quad$ Szeged & 996 & 32 & 3.2 \\
Budapest I & 187 & 15 & 8.0 \\
Budapest II & 674 & 20 & 3.0 \\
Szombathely & 289 & 6 & 2.1 \\
Eastern region (high rate of unemployment) & 4010 & 289 & 7.2 \\
$\quad$ Debrecen & 473 & 6 & 1.3 \\
$\quad$ Miskolc & 2112 & 208 & 9.8 \\
$\quad$ Nyíregyháza & 1416 & 75 & 5.3 \\
\hline Total & 6156 & 362 & 5.9 \\
\hline
\end{tabular}


Table II. Risk factors for C. trachomatis infections among 6156 pregnant women

\begin{tabular}{|c|c|c|c|c|c|}
\hline Risk factors & $\mathrm{N}$ & $\begin{array}{l}\text { Number of } \\
\text { C. tracho- } \\
\text { matis- } \\
\text { infections }\end{array}$ & $\begin{array}{l}\text { Rate of } \\
\text { chlamydial } \\
\text { infection } \\
(\%)\end{array}$ & $\begin{array}{l}\text { Odds ratio } \\
(95 \% \mathrm{CI})\end{array}$ & p value* \\
\hline Age & & & & & $\mathrm{p}=0.0002$ \\
\hline$<=24$ years & 2469 & 196 & 7.9 & $1.6(1.3-2.0)$ & \\
\hline$>24$ years & 3687 & 166 & 4.5 & 1.0 & \\
\hline Family status & & & & & $\mathrm{p}=0.0014$ \\
\hline unmarried & 1152 & 98 & 8.5 & $1.5(1.2-1.9)$ & \\
\hline married & 5004 & 264 & 5.3 & 1.0 & \\
\hline Rate of unemployment & & & & & $\mathrm{p}=0.0001$ \\
\hline high & 4010 & 289 & 7.2 & $2.1(1.6-2.7)$ & \\
\hline normal & 2146 & 73 & 3.4 & 1.0 & \\
\hline
\end{tabular}

* Adjusted odds ratios and $\mathrm{p}$ values from multivariate logistic regression analysis. The Hosmer and Lemeshow statistics to assess model goodness of fit had a value of 5.47 with $4 \mathrm{df}(\mathrm{p}=0.24)$. Thus this measure supports the model's adequacy for the data.

group of $C$. trachomatis-positive patients than in the group of negative patients $(2.3 \%)$. In the group of 148 perinatal deaths, the maternal $C$. trachomatis infection rate was $10.1 \%$.

With regard to the $C$. trachomatis infection and perinatal mortality rates in the normal and low birth weight groups, among the 28 infants under $2500 \mathrm{~g}$ the maternal $C$. trachomatis infection rate was $21.4 \%$, whereas in the group of 120 infants above $2500 \mathrm{~g}$ the maternal infection rate was only $7.5 \%$. This difference was significant $(\mathrm{p}=0.044)$.

In the multiple logistic regression analysis some potential risk factors of perinatal mortality were examined: age, demographic and social status, C. trachomatis infection, previous pregnancy, previous delivery, low birth weight, premature rupture of membrane, premature uterine activity, intrauterine distress and intrauterine growth retardation of the neonate. As shown in table III. C. trachomatis infection (OR and $95 \%$ CI: $1.9(1.1-3.3))$, low birth weight $(1.7(1.1-$ $2.7)$, no previous delivery $(1.9(1.3-2.7)$ and the high unemployment rate $(1.5(1.2-2.2))$ were all significant predictors of the neonatal mortality $(p<0.05)$ in the multiple regression analysis.

\section{Discussion}

Numerous surveys have been carried out to study the prevalence of urogenital $C$. trachomatis infections. It is necessary to estimate the true prevalence of $C$. trachomatis infection because detection and treatment of asymptomatic cases should have a major impact on the incidence of chlamydial infection, as these asymptomatic individuals are an active source of new infections. Precise estimation of the prevalence of $C$. trachomatis infection is important in cost-effectiveness analysis [15]. In developed countries, the debate on Chlamydia control has centered on the cost-effectiveness of different approaches for identifying infected persons. Studies have concluded that, among women, case findings using laboratory tests is cost-effective at $C$. trachomatis prevalence rates as different as $6 \%$ to $16 \%$ [13] as Sellors et al found. In epidemiology, some methods have been suggested to correct for measurement error. In our analyses a Bayesian approach was employed for correcting measurement errors $[2,12]$. In our study the observed overall rate of C. trachomatis infection in Hungary was $5.9 \%$, increased to $7.1 \%$ based on the Bayesian approach. In the eastern region, where the rate of unemployment is high the prevalence is estimated to be $7.2 \%$. 
Table III. Risk factors for perinatal mortality in pregnancies screened for C. trachomatis

\begin{tabular}{|c|c|c|c|c|c|}
\hline Risk factors & $\mathrm{N}$ & $\begin{array}{l}\text { Number of } \\
\text { perinatal } \\
\text { mortality } \\
\text { outcome }\end{array}$ & $\begin{array}{l}\text { Rate of } \\
\text { mortality } \\
(\%)\end{array}$ & $\begin{array}{l}\text { Odds ratio } \\
(95 \% \mathrm{CI})\end{array}$ & $\mathrm{p}$ value* \\
\hline C. trachomatis infection & & & & & $\mathrm{p}=0.033$ \\
\hline infected & 362 & 15 & 4.1 & $1.8(1.1-3.3)$ & \\
\hline non infected & 5794 & 133 & 2.3 & 1.0 & \\
\hline Low birth weight & & & & & $\mathrm{p}=0.020$ \\
\hline Yes & 645 & 24 & 3.7 & $1.7(1.1-2.7)$ & \\
\hline No & 5400 & 120 & 2.2 & 1.0 & \\
\hline Previous delivery & & & & & $\mathrm{p}=0.001$ \\
\hline no previous delivery & 3219 & 100 & 3.1 & $1.9(1.3-2.7)$ & \\
\hline previous delivery & 2937 & 48 & 1.6 & 1.0 & \\
\hline Rate of unemployment & & & & & $\mathrm{p}=0.010$ \\
\hline high & 4010 & 108 & 2.7 & $1.5(1.1-2.2)$ & \\
\hline normal & 2146 & 40 & 1.9 & 1.0 & \\
\hline
\end{tabular}

* Adjusted odds ratios and $\mathrm{p}$ values from multiple logistic regression analysis. (Data missing up to 111 women) The Hosmer and Lemeshow statistics to assess model goodness of fit has a value of 4.058 with $5 \mathrm{df}(\mathrm{p}=0.54)$. This measure supports the model's adequacy for the data.

The perinatal mortality rate is one of the most important indicators of the quality of perinatal care. As with [7] Ngassa and [9] Orfila et al., our results suggest that C. trachomatis infection is of great importance in perinatal pathologies. The perinatal mortality rate was significantly higher in the $C$. trachomatis positive patients. The difference was more marked in the low birth weight group than in the normal birth weight cases. In the multiple regression analysis the C. trachomatis infection, low birth weight, no previous delivery and the high unemployment rate were independent statistically significant predictors of neonatal mortality.

\section{Abstract}

Introduction: Chlamydial infections of the genital tract are thought to often lead to preterm birth, which is the most important perinatal problem in Hungary.

Aim of study: A multicenter study was carried out to determine the prevalence of Chlamydia trachomatis infection, risk factors for the infection and to relate the infection to perinatal mortality, accounting for potential confounding effects.

Methods: The nucleic acid hybridization method (PACE2 Gen-Probe) was applied for the examination of
In summary, testing pregnant women for diseases that can be transmitted perinatally is an important part of obstetric care. Screening for C. trachomatis is suggested only in high-risk pregnancies such as those involving an unfavorable obstetric history, a poor socio-economic situation and a young age less than 24 years old. Any attempt to prevent the spread of STDs must include the provision of contraceptives, sex education, efforts to arouse a more responsible attitude to pregnancy, and attempts to improve the social environment [1].

Chlamydia trachomatis. Logistic regression analysis was used to assess risk.

Results: A total of 6156 pregnant women were examined for the occurrence of Chlamydia trachomatis. The observed overall rate of chlamydial infection was $5.9 \%$. Young age (less than 24 years old) (OR and 95\% CI:1.6 $(1.3-2.0))$, unmarried status $(1.5(1.2-1.9))$ and the high unemployment rate $(2.1(1.6-2.7))$ were statistically significant predictors of the infection. 
In logistic regression analysis, chlamydial infection (1.9 $(1.1-3.3))$, high unemployment rate $(1.5(1.2-2.2))$ and low birth weight $(1.7(1.1-2.7)$ were significant predictors of perinatal mortality.
Conclusions: Testing pregnant women for diseases that can be transmitted perinatally is an important part of obstetric care. Screening for C. trachomatis of unmarried women under 24 years of age is suggested and need increased observation during labor.

Keywords: Chlamydia trachomatis, perinatal mortality, poor perinatal outcome, preterm birth, preventive care.

\section{References}

[1] Ancel P-Y, MJ Saurel-Cubizolles, GC Di Renzo, E Papiernik, G Breart and the Europop Group: Social differences of very preterm birth in Europe: Interaction with obstetric history. Am J Epidem 149 (1999) 908

[2] Bashir SA, SW Duffy: Correction of risk estimates for measurement error in epidemiology. Meth Inform Med 34 (1995) 503

[3] Czeizel AE, M. Rockenbauer: A lower rate of preterm birth after clotrimazole therapy during pregnancy. Paediatr Perinat Epidemiol 13 (1999) 58

[4] Hsieh FY: Sample size tables for logistic regression. Stat Med 8 (1989) 795

[5] Muh DH, SY Yeh: Relevance of Chlamydia trachomatis infection in pregnant patients. Public Health Rep 106 (1991) 490

[6] Newhall WJ, RE Johnson, S Delisle, D Fine, A Hadgu, B Matsuda, D Osmond, J Campbell, WE Stamm: Head-to-head evaluation of five chlamydia tests relative to a quality-assured culture standard. J Clin Microbiol 37 (1999) 681

[7] Ngassa PC, JA Egbe: Maternal genital Chlamydia trachomatis infection and the risk of preterm labour. Int J Gynecol Obstet 57 (1994) 241

[8] Nyári T, J Deák, E Nagy, I Veréb, L Kovács, GY Mészáros, H Orvos, I Berbik: Epidemiological study of Chlamydia trachomatis infection in pregnant women in Hungary. Sex Transm Inf 74 (1998) 213

[9] Orfila J, P Beque, C Ruggeri, T Mathieu, Y Adjuard, JC Borderon: Incidence of nonbacterial intraamniotic infections in abnormal pregnancies. J Reprod Med 36 (1991) 783
[10] Orvos H, I Nyirati, J Hajdú, A Pál, T Nyári, L Kovács: Is adolescent pregnancy associated with adverse perinatal outcome? J Perinat Med 27 (1999) 199

[11] Richardson S, WR Gilks: A Bayesian appproach to measurement error problems in epidemiology using conditional independence models. Am J Epidem 138 (1993) 430

[12] Rogan WJ, B Gladen: Estimating prevalence from the results of a screening test. Am J Epidem 107 (1978) 71

[13] Sellors JW, L Pickard, A Gafni, CH Goldsmith, D Jang, JB Mahony, MA Chernesky: Effectiveness and efficiency of selective vs universal screening for chlamydial infection in sexually active young women. Arch Intern Med 152 (1992) 1837

[14] Sweet RL, DV Landers, C Walker, J Schachter: Chlamydia trachomatis infection and pregnancy outcome. Am J Obstet Gynecol 156 (1987) 824

[15] Trachtenberg AI, AE Washington, S Halldorson: A cost-based decision analysis for Chlamydia screening in California family planning clinics. Obstet-Gynecol 71 (1988) 101

Received June 7, 2000. Revised August 2, 2000. Accepted August 16, 2000.

Tibor Nyári, Ph. D.

Department of Medical Informatics

University of Szeged

H-672O Szeged, P. O. Box:427, Hungary

Tel./Fax: +36-62/544-566

e-mail: Nyari@dmi.szote.u-szeged.hu 